\title{
EXTREME POINT METHODS AND BANACH-STONE THEOREMS
}

\author{
HASAN AL-HALEES and RICHARD J. FLEMING
}

(Received 3 December 2001; revised 3 June 2002)

Communicated by A. Pryde

\begin{abstract}
An operator is said to be nice if its conjugate maps extreme points of the dual unit ball to extreme points. The classical Banach-Stone Theorem says that an isometry from a space of continuous functions on a compact Hausdorff space onto another such space is a weighted composition operator. One common proof of this result uses the fact that an isometry is a nice operator. We use extreme point methods and the notion of centralizer to characterize nice operators as operator weighted compositions on subspaces of spaces of continuous functions with values in a Banach space. Previous characterizations of isometries from a subspace $M$ of $C_{0}(Q, X)$ into $C_{0}(K, Y)$ require $Y$ to be strictly convex, but we are able to obtain some results without that assumption. Important use is made of a vector-valued version of the Choquet Boundary. We also characterize nice operators from one function module to another.
\end{abstract}

2000 Mathematics subject classification: primary 46B04, 46E40.

\section{Introduction}

The classical Banach-Stone theorem says that if $T$ is an isometry from the space $C(Q)$ of continuous functions on a compact Hausdorff space $Q$ onto $C(K)$, where $K$ is a compact Hausdorff space, then there is a continuous function $h$ on $K$ of modulus one, and a homeomorphism $\varphi$ from $K$ onto $Q$ such that

$$
T f(t)=h(t) f(\varphi(t))
$$

for all $f \in C(Q)$ and $t \in K$. There are many proofs of this theorem existing in the literature, and one of the best known proof (as given by Dunford and Schwartz [8], for example) uses the fact that the conjugate of an isometry must map extreme points of the unit ball of the dual space of $C(K)$ onto the extreme points of the unit ball of the

(C) 2003 Australian Mathematical Society $1446-7887 / 03 \$$ A2.00+0.00 
dual space of $C(Q)$. Proved first by Banach [2] for the real compact metric case and later by Stone [19] in the general real case, the theorem has inspired numerous articles dealing with different proof techniques and a variety of generalizations. Behrends [3] has given an excellent account of these matters, and the reader might also consult [9] for some of the history.

One stream of investigation has focused on the case where the continuous functions have values in a Banach space $E$ and the question to be answered is whether the operator $T$ can be described by some kind of canonical form as is given in (1). The first such result along these lines was given by Jerison [13]. Many others followed, and we state below a theorem of Behrends [3, Theorem 8.10] which includes many of the earlier results.

THEOREM 1.1. Let $E_{1}$ and $E_{2}$ be Banach spaces which have one dimensional centralizers $Z\left(E_{1}\right), Z\left(E_{2}\right)$, respectively. Suppose further, that $Q$ and $K$ are locally compact Hausdorff spaces and that there exists an isometric isomorphism $T$ from $C_{0}\left(Q, E_{1}\right)$ onto $C_{0}\left(K, E_{2}\right)$. Then there is a homeomorphism $\varphi$ from $K$ onto $Q$ and $a$ continuous function $V$ from $K$ into the space of isometries from $E_{1}$ to $E_{2}$ (given the strong operator topology) such that

$$
T F(t)=V(t) F(\varphi(t))
$$

for all $t \in K$ and $F \in C_{0}\left(Q, E_{1}\right)$.

Our principal goal in this paper is to characterize isometries $T$ as in the theorem above, but without assuming that $T$ is surjective, or even defined on all of $C_{0}\left(Q, E_{1}\right)$. One of the first to consider non-surjective isometries from $C(Q)$ into $C(K)$ was Holsztyński [11], who showed that one could get a canonical form, but the function $\varphi$ may not be defined on all of $K$. Cambern [6] extended this to the vector valued case where $E$ is assumed to be strictly convex. Novinger [17] considered isometries from certain subspaces $M$ of $C_{0}(Q)$ onto a subspace $N$ of $C_{0}(K)$, and Font [10] has studied isometries of this type for vector-valued functions. Font's approach is similar to that of Cambern, while Novinger used extreme point techniques and the notion of Choquet Boundary.

The characterization of an operator as in (2) above involves getting the function $\varphi$ defined in some manner. Our method will be to use the fact that the conjugate of an isometry maps extreme points to extreme points. In order to emphasize this property, we will consider nice operators.

An operator is said to be nice if its conjugate takes extreme points to extreme points. An isometry is an example of a nice operator. A key role is played by the concept of centralizer of a Banach space. This will be defined in Section 2, where we will also prove a version for nice operators of Theorem 1.1 stated above. We treat the surjective 
case first in order to establish our methods in a more hospitable climate. In Section 3 we examine the situation in which the operator $T$ maps a subspace $M$ of $C_{0}\left(Q, E_{1}\right)$ onto a subspace $N$ of $C_{0}\left(K, E_{2}\right)$ but without assuming that $E_{2}$ is strictly convex. It is necessary to put some special conditions on both $M$ and $N$. Finally, in Section 4, we consider the special case in which the space $E_{2}$ is strictly convex.

It should be mentioned that many of the results in this paper are contained in the Ph.D. dissertation of the first author [1].

\section{Centralizers, function modules, and Behrends' Theorem}

In what follows, $Q, K$ denote locally compact Hausdorff spaces and $E, E_{1}, E_{2}$, $X, Y$ represent Banach spaces. As usual, $C_{0}(K, E)$ will mean the Banach space of continuous $E$-valued functions defined on $K$ and vanishing at infinity. If $F \in$ $C_{0}(K, E)$, then $\|F\|=\|F\|_{\infty}=\sup _{t \in K}\|F(t)\|$. We will assume that the scalar field $\mathbb{F}$ is always the complex numbers $\mathbb{C}$ unless specifically noted to be $\mathbb{R}$. In case $E$ is the scalar field, we will write $C_{0}(K, E)$ as simply $C_{0}(K)$. (Of course, if $K$ is compact, we omit the sub-zero.) For Banach spaces $X$ and $Y$, we will let $\mathscr{L}(X, Y)$ denote the bounded linear operators from $X$ to $Y$, and if $T \in \mathscr{L}(X, Y)$, then $T^{*}$ is the usual conjugate of $T$ defined by $T^{*} y^{*}(x)=y^{*}(T x)$. By $B(X)$ we will mean the closed unit ball of $X, S(X)$ will be the unit sphere, and $\operatorname{ext}(A)$ will be the set of extreme points of $A$. In the particular case that $A$ is a Banach space, we will write $\operatorname{ext}(A)$ in place of $\operatorname{ext}(B(A))$. By $A^{-}$we will mean the closure of $A$. For $1 \leq p \leq \infty, \quad \ell^{p}(n)$ will denote the $n$-dimensional $\ell^{p}$ space.

DEFINITION 2.1. Let $T$ be a bounded linear operator on $X$.

(i) The operator $T$ is a multiplier of $X$ if every element of $\operatorname{ext}\left(X^{*}\right)$ is an eigenvector for $T^{*}$. Hence for each $x^{*} \in \operatorname{ext}\left(X^{*}\right)$ we have a scalar $a_{T}\left(x^{*}\right)$ such that

$$
T^{*} x^{*}=a_{T}\left(x^{*}\right) x^{*} .
$$

(ii) The operator $T$ is said to be $M$-bounded if there is a $\lambda>0$ such that, for every $x \in X, T x$ is contained in every ball which contains $\{\mu x: \mu \in \mathbb{F},|\mu| \leq \lambda\}$.

(iii) For a multiplier $T$ on $X$, we say that a multiplier $S$ on $X$ is an adjoint for $T$ if $a_{S}=\overline{a_{T}}$. If $T$ has an adjoint, we will denote it by $T^{a}$.

(iv) The centralizer of $X$, written as $Z(X)$, is the set of all multipliers for which an adjoint exists. (Note that in case $\mathbb{F}=\mathbb{R}$, the centralizer just consists of the multipliers.)

Definition 2.2. A function module is a triple $\left(K,\left(X_{t}\right)_{t \in K}, X\right)$, where $K$ is a nonvoid compact Hausdorff space (the base space), $\left(X_{t}\right)_{t \in K}$ a family of Banach spaces (the component spaces), and $X$ a closed subspace of $\prod_{t \in K}^{\infty} X_{t}$ such that the following conditions are satisfied 
(i) $h x \in X$ for $x \in X$ and $h \in C(K)((h x)(t)=h(t) x(t))$;

(ii) $t \rightarrow\|x(t)\|$ is an upper semicontinuous function for every $x \in X$;

(iii) $X_{t}=\{x(t): x \in X\}$ for every $t \in K$;

(iv) $\left\{t: t \in K, X_{t} \neq\{0\}\right\}^{-}=K$.

Note: The space $\prod_{t \in K}^{\infty} X_{t}$ denotes the functions $x$ in the product space for which $\|x\|=\sup \{\|x(t)\|: t \in K\}<\infty$.

The above definitions along with their history and many properties may be found in [3]. Of particular interest to us is the fact that $T$ is a multiplier if and only if it is $M$-bounded. A natural example of a function module is a space $C(K, E)$ where we take $X_{t}=E$ for each $t \in K$. In the locally compact case, we can replace $K$ by its Stone-Cêch compactification $\beta K$ and take $X_{t}=E$ if $t \in K$ and $X_{t}=\{0\}$ if $t \in \beta K \backslash K$.

A property of function modules that will be of importance later is that a function module is norming. We will say that a function space $M$ with functions defined on a set $Q$ and values in $E$ is norming for $Q$ if for each $s \in Q$ and $u \in E$ there is some $F \in M$ such that $F(s)=u$ and $\|F(s)\|=\|F\|$.

LEMMA 2.3. If $\left(K,\left(X_{t}\right)_{t \in K}, X\right)$ is a function module and $t \in K, u \in X_{t}$ are given, there exists $F \in X$ such that $F(t)=u$ and $\|F\|=\|F(t)\|=\|u\|$. Furthermore, if $U$ is a neighbourhood of $t, F$ may be chosen as above so that $F(r)=0$ for $r \in K \backslash U$.

PROOF. This follows directly from [3, Lemma 4.2].

A function module representation $\left[\rho,\left(K,\left(X_{t}\right)_{t \in K}, \tilde{X}\right)\right]$ of a Banach space $X$ is a function module $\left(K,\left(X_{t}\right)_{t \in K}, \tilde{X}\right)$ together with an isometric isomorphism $\rho: X \rightarrow \tilde{X}$. If for $h \in C(K)$ we let $M_{h}$ be the multiplication operator on $\tilde{X}$ defined by $M_{h} x(t)=$ $h(t) x(t)$, then $Z_{\rho}(X)=\left\{\rho^{-1} M_{h} \rho: h \in C(K)\right\}$ is contained in $Z(X)$. It is known that for any Banach space $X$ there is a function module representation of $X$ as above such that $Z_{\rho}(X)=Z(X)$ [3]. We will not use this idea directly in the current paper. It is easy to see that for $h \in C(K), M_{h}$ is a multiplier and $M_{\bar{h}}$ is its adjoint, and, in fact, $Z(C(K))=\left\{M_{h}: h \in C(K)\right\}$. In the locally compact case, the centralizer consists of multiplications by elements of $C^{b}(K)$, the bounded continuous functions on $K$. It is natural to ask about the centralizer of $C_{0}(K, E)$.

Before doing anything else, we want to state the following well-known fact about extreme points. The proof is straightforward and we omit it. However, the result will be used many times in the remainder of the paper.

LEMMA 2.4. For a given $x$ in a Banach space $X$, there exists $x^{*} \in \operatorname{ext}\left(X^{*}\right)$ such that $x^{*}(x)=\|x\|$. 
The extreme points of the unit ball of $C_{0}(K, E)^{*}$ are known to be of the form $x^{*} \circ \psi_{t}$ where $x^{*} \in \operatorname{ext}\left(E^{*}\right)$ and $\psi_{t}$ is the evaluation functional at $t \in K[3,4]$. If $h \in C_{0}(K)$, then $M_{h}^{*}\left(x^{*} \circ \psi_{t}\right)=h(t)\left(x^{*} \circ \psi_{t}\right)$ so that $M_{h}$ is a multiplier with adjoint $M_{\bar{h}}$. Hence $M_{h}$ is in the centralizer of $C_{0}(K, E)$. Behrends [3] has shown that these operators describe the centralizer entirely under a certain condition on $E$. We will say that $E$ has trivial centralizer if $Z(E)$ has dimension 1 , that is, if the only elements in the centralizer are multiples of the identity operator. We are first going to describe the centralizer of a Banach space $X$ which is, itself, a function module. We will need the fact, proved in [3, Theorem 4.5], that the extreme points of the unit ball of $\left(K,\left(X_{t}\right)_{t \in K}, X\right)$ are precisely those elements of the form $x^{*} \circ \psi_{t}$ where $x^{*} \in \operatorname{ext}\left(X_{t}^{*}\right)$. A proof of the next lemma can also be found in [3], but we give one here in order to show clearly the importance of the assumptions about a trivial centralizer.

LEMMA 2.5. Let $\left(K,\left(X_{t}\right)_{t \in K}, X\right)$ be a function module with the property that $Z\left(X_{t}\right)$ is trivial for each $t \in K$. Then $Z(X)=\left\{M_{h}: h\right.$ is bounded on $K$ and $h F \in X$ for all $F \in X\}$.

Proof. If $h$ is a bounded scalar-valued function on $K$ such that $h F \in X$ for all $F \in X$, then it is straightforward to show $M_{h}$ is in the centralizer of $X$. Suppose, on the other hand, that $W \in Z(X)$. Given $t \in K_{0}=\left\{t \in K: X_{t} \neq\{0\}\right\}$ and $x^{*} \in \operatorname{ext}\left(X_{t}^{*}\right)$ we have a scalar $a_{W}\left(x^{*}, t\right)$ such that $W^{*}\left(x^{*} \circ \psi_{t}\right)=a_{W}\left(x^{*}, t\right)\left(x^{*} \circ \psi_{t}\right)$. Note that

$$
\left|a_{W}\left(x^{*}, t\right)\right|=\left\|a_{W}\left(x^{*}, t\right)\left(x^{*} \circ \psi_{t}\right)\right\|=\left\|W^{*}\left(x^{*} \circ \psi_{t}\right)\right\| \leq\left\|W^{*}\right\|=\|W\| .
$$

Let us define $P(t)$ on $X$, by $P(t) u=W F(t)$, where $F \in X$ with $F(t)=u$. Now $P(t)$ is well defined, for if $F(t)=H(t)$, then

$$
\begin{aligned}
x^{*}(W F(t)) & =a_{W}\left(x^{*}, t\right) x^{*}(F(t))=a_{W}\left(x^{*}, t\right) x^{*}(H(t)) \\
& =a_{W}\left(x^{*}, t\right)\left(x^{*} \circ \psi_{t}\right)(H)=W^{*}\left(x^{*} \circ \psi_{t}\right)(H)=x^{*}(W H(t))
\end{aligned}
$$

for all $x^{*} \in \operatorname{ext}\left(X_{t}^{*}\right)$. Hence, $W F(t)=W H(t)$. Moreover, $P(t)$ is bounded, for if $u \in X_{t}$, and $F(t)=u$, by Lemma 2.4 there exists $x^{*} \in \operatorname{ext}\left(X_{t}^{*}\right)$ such that $x^{*}(W F(t))=\|W F(t)\|$. Thus, from (3) we obtain

$$
\begin{aligned}
\|P(t) u\| & =\|W F(t)\|=W^{*}\left(x^{*} \circ \psi_{t}\right)(F) \\
& =\left|a_{W}\left(x^{*}, t\right)\left(x^{*} \circ \psi_{t}\right)(F)\right| \leq\left|a_{W}\left(x^{*}, t\right)\right|\|u\| \leq\|W\|\|u\| .
\end{aligned}
$$

Similar manipulations show that $P^{*}(t) x^{*}=a_{W}\left(x^{*}, t\right) x^{*}$, and $P(t)$ is a multiplier. Since it will have an adjoint obtained from the adjoint $W^{a}$ of $W$, we conclude that $P(t) \in Z\left(X_{t}^{*}\right)$. Consequently, there is a scalar $h(t)$ such that $P(t)=h(t) I$ and we have $W F(t)=h(t) F(t)$ for all $t \in K_{0}$, where $h(t)=a_{W}\left(x^{*}, t\right)$ for all $x^{*}$. That $h$ is bounded on $K_{0}$ follows from (3). It does not matter what values $h$ is given outside $K_{0}$; they can be any finite constant. 
The corollary below was observed by Behrends in his proof of Theorem 8.10 in [3].

COROLlARY 2.6. Let $K$ be a locally compact Hausdorff space and $E$ a Banach space with trivial centralizer. Then $Z\left(C_{0}(K, E)\right)=\left\{M_{h}: h \in C^{b}(K)\right\}$.

This corollary follows immediately from Lemma 2.5 since $C_{0}(K, E)$ is a function module. The continuity of $h$ is easy to show using the fact that there is a function $F$ that is constant on some neighbourhood of a given $t$.

Definition 2.7. An operator $T \in \mathscr{L}(X, Y)$ is said to be nice if

$$
T^{*}\left(\operatorname{ext}\left(Y^{*}\right)\right) \subseteq \operatorname{ext}\left(X^{*}\right)
$$

This notion is apparently due to Morris and Phelps [16] and is a sufficient (but not necessary) condition for $T$ to be an extreme point of the unit ball of $\mathscr{L}(X, Y)$. Every isometry is nice, but not every nice operator, nor even a nice isomorphism, is necessarily an isometry. Clearly, if both $T$ and $T^{-1}$ are nice, then $T$ is an isometry.

Werner [20] has observed that a nice operator from $C(Q)$ to $C(K)$ can be described as a weighted composition operator as given by (1). We are going to extend that result to the vector-valued case. First we show that a nice isomorphism from one function module to another has the canonical form (2).

Note that a nice operator is necessarily a contraction. For, if $x \in X$ and if $y^{*} \in \operatorname{ext}\left(Y^{*}\right)$ so that $\left|y^{*}(T x)\right|=\|T x\|$, then

$$
\|T x\|=\left|y^{*}(T x)\right|=\left|T^{*} y^{*}(x)\right| \leq\|x\| .
$$

THEOREM 2.8. Suppose $T$ is a nice isomorphism from the function module $X=$ $\left(Q,\left(X_{s}\right)_{s \in Q}, X\right)$ onto the function module $\left(K,\left(Y_{t}\right)_{t \in K}, Y\right)$ where $Z\left(Y_{t}\right)$ is trivial for each $t \in K$ such that $Y_{t} \neq\{0\}$. Then there is a function $\varphi$ from $K_{0}=\left\{t \in K: Y_{t} \neq 0\right\}$ onto a dense subset of $Q$ and a function $t \rightarrow V(t)$ from $K_{0}$ into the family of nice operators from $X_{\varphi(t)}$ to $Y_{t}$ such that

$$
T F(t)=V(t) F(\varphi(t))
$$

for all $t \in K_{0}$ and $F \in X$.

Proof. Let $h$ be a continuous function on $Q$. We will first show that $T M_{h} T^{-1}$ is in the centralizer of $Y$. If $t \in K_{0}, y^{*} \in \operatorname{ext}\left(Y_{t}^{*}\right)$, then, since $T$ is nice, there are $s \in Q$ and $x^{*} \in \operatorname{ext}\left(X_{s}^{*}\right)$ such that $T^{*}\left(y^{*} \circ \psi_{l}\right)=x^{*} \circ \psi_{s}$. Hence, for $G=T F \in Y$, we have (after a bit of manipulation),

$$
\left(T M_{h} T^{-1}\right)^{*}\left(y^{*} \circ \psi_{t}\right)(G)=h(s)\left(y^{*} \circ \psi_{t}\right)(G) .
$$


This shows that each extreme point of $S\left(Y^{*}\right)$ is an eigenvector and so $T M_{h} T^{-1}$ is a multiplier. Its adjoint will be given by $T M_{\bar{h}} T^{-1}$ and so $T M_{h} T^{-1}$ is in the centralizer. By Lemma 2.5, there is a bounded function $\tilde{h}$ on $K$ such that $T M_{h} T^{-1}=M_{\bar{h}}$. This implies that $T M_{h}=M_{\tilde{h}} T$. It is easy to show that

$$
h(s) x^{*}(F(s))=\left(T M_{h}\right)^{*}\left(y^{*} \circ \psi_{t}\right)(F)=\left(M_{\tilde{h}}\right)^{*}\left(y^{*} \circ \psi_{t}\right)(T F)=\tilde{h}(t) x^{*}(F(s))
$$

for any $F \in X$. Hence, we must conclude that $h(s)=\tilde{h}(t)$. Now, if we have $y^{*}, z^{*} \in \operatorname{ext}\left(Y_{t}^{*}\right)$ and

$$
T^{*}\left(y^{*} \circ \psi_{t}\right)=x^{*} \circ \psi_{s} \text { and } T^{*}\left(z^{*} \circ \psi_{t}\right)=w^{*} \circ \psi_{r},
$$

it follows that $h(s)=\tilde{h}(t)=h(r)$. Since there are enough continuous functions on $Q$ to separate points of $Q$, we must have $r=s$. We define the function $\varphi$ on $K_{0}$ by $\varphi(t)=s$ according to the pairing determined by $T^{*}$.

Next we define, for each $t \in K_{0}$ an operator $V(t)$ on $X_{s}=X_{\varphi(t)}$ by $V(t) u=T F(t)$, where $F \in X$ has the property that $F(\varphi(t))=u$. To see that $V(t)$ is well defined, suppose that $s=\varphi(t)$ and $F(s)=H(s)$. Then for any extreme point $y^{*}$ for $B\left(Y_{t}^{*}\right)$, and $T^{*}\left(y^{*} \circ \psi_{t}\right)=x^{*} \circ \psi_{s}$ we have

$$
y^{*}(T F(t))=x^{*}(F(s))=x^{*}(H(s))=y^{*}(T H(t)),
$$

from which it follows that $T F(t)=T H(t)$. Let $u \in X_{\varphi(t)}$ be given and $F \in X$ so that $F(\varphi(t))=u$. There exists $y^{*} \in \operatorname{ext}\left(Y_{t}^{*}\right)$ with $\|V(t) u\|=\|T F(t)\|=y^{*}(T F(t)$. Hence, $\|V(t) u\|=y^{*}\left(T F(t)=T^{*}\left(y^{*} \circ \psi_{t}\right)(F)=x^{*}(F(\varphi(t)) \leq\|u\|\right.$. Thus $V(t)$ is bounded and nice, since $V(t)^{*}$ maps extreme points of $Y_{t}^{*}$ to extreme points of $X_{\varphi(t)}^{*}$. Hence we have established (4), for by its definition, $V(t) F(\varphi(t))=T F(t)$.

To complete the proof, we show that $\varphi\left(K_{0}\right)$ is dense in $Q$. Suppose $s_{0}$ is such that $X_{s_{0}} \neq 0$ and $s_{0} \in Q \backslash \varphi\left(K_{0}\right)^{-}$. By Lemma 2.3 there exists $F \in X$ so that $F\left(s_{0}\right) \neq 0$ but $F(r)=0$ for all $r \in \varphi\left(K_{0}\right)^{-}$. Thus $T F(t)=V(t) F(\varphi(t))=0$ for all $t \in K_{0}$. This contradicts the fact that $T$ is injective, and from part (iv) of Definition 2.2, we conclude that $\varphi\left(K_{0}\right)$ is dense.

We want to note here that Behrends [3, Theorem 4.16, Corollary 4.17] proves the above theorem for $T$ an isometric isomorphism and under the assumption that $Z(X)=\left\{M_{h}: h \in C(Q)\right\}$ and $Z(Y)=\left\{M_{h}: h \in C(K)\right\}$. In that case, the function $\varphi$ is proved to be continuous, since $T M_{h} T^{-1}$ induces an isometric isomorphism $\omega$ from $C(Q)$ onto a closed self-adjoint subalgebra of $C(K)$. Hence $\omega(h)=h \circ \varphi$ for some continuous function $\varphi$ by classical results. In our case we are unable to show that $\varphi$, as we defined it, is necessarily continuous. Indeed, it need not be continuous. For example, consider the subspace $X$ of the bounded scalar-valued functions on $[0,1]$ where $X=\{f:\{t:|f(t)| \geq \epsilon\}$ is finite for every $\epsilon>0\}$. Then $X$ is a Banach 
function module [3, page 78] and if $\varphi$ is a one-to-one function from $[0,1]$ onto $[0,1]$, then $T f=f \circ \varphi$ is an isometry from $X$ onto $X$. However, $\varphi$ could be taken to be discontinuous on $[0,1]$.

THEOREM 2.9. Suppose $T$ is a nice isomorphism from $C_{0}\left(Q, E_{1}\right)$ onto $C_{0}\left(K, E_{2}\right)$ and suppose that $E_{2}$ has a trivial centralizer. Then there exists a continuous function $\varphi$ from $K$ onto a dense subset of $Q$ and a continuous function $t \rightarrow V(t)$ from $K$ into the collection of nice operators contained in $\mathscr{L}\left(E_{1}, E_{2}\right)$ (given the strong operator topology) such that $T F(t)=V(t) F(\varphi(t))$ for all $t \in K$ and $F \in C_{0}\left(Q, E_{1}\right)$.

PROOF. The canonical form comes immediately from Theorem 2.8 , since the spaces in question are function modules as described in the paragraph following Definition 2.2. Once again, $K$ plays the role of $K_{0}$ in that theorem.

It remains to prove the continuity assertions. Suppose the function $\varphi$ is not continuous at some $t \in K$. Then there is a net $\left\{t_{\beta}\right\}$ in $K$ converging to $t$ and a neighbourhood $U$ of $s=\varphi(t)$ such that for every $\beta_{0}$ there exists $\beta \geq \beta_{0}$ with $\varphi\left(t_{\beta}\right) \in Q \backslash U$. Let $u \in E_{1}$ be such that $\|V(t) u\|=1$. By Lemma 2.3, there is an $F \in C_{0}\left(Q, E_{1}\right)$ such that $F(s)=u,\|F\|=\|u\|$, and $F(r)=0$ for $r \in Q \backslash U$. Now we have $\|T F(t)\|=\|V(t) u\|=1$, and by the continuity of $T F$ and the choice of $U$ and $\left\{t_{\beta}\right\}$ we must have $1=\|T F(t)\|=\liminf \left\|T F\left(t_{\beta}\right)\right\|=0$. This contradiction establishes the continuity of $\varphi$.

The continuity of $t \rightarrow V(t)$ comes immediately from the facts that for a given $u \in E_{1}$, and $t \in K$, there is a function $F \in C_{0}\left(Q, E_{1}\right)$ which is constantly equal to $u$ on a compact neighbourhood of $\varphi(t)$ and $T F$ is continuous at $t$.

REMARKS. (i) The crucial part of the argument for the theorems above is the establishment of the function $\varphi$. The pairing of $t$ with $s$ is easy using the extreme points but the chief difficulty is in showing that $\varphi$ is well-defined; that is, showing that statement (5) implies that $s=r$. It is here that the hypothesis about $Z\left(E_{2}\right)$ is needed. Examples show that some such requirement is necessary [5].

(ii) The condition that $\operatorname{dim}\left(Z\left(E_{2}\right)\right)=1$ is not as restrictive as it might appear. Strictly convex spaces, smooth spaces, and reflexive spaces with no M-summand all have this property [3].

(iii) If $T^{-1}$ is also nice, so that $T^{*}$ maps $\operatorname{ext}\left(Y^{*}\right)$ onto $\operatorname{ext}\left(X^{*}\right)$, then $\varphi$ maps $K$ onto $Q$. In this case, of course, $T$ is an isometry.

(iv) In Theorem 2.9, if $K$ is compact, then $\varphi$ is surjective so that $Q$ is also compact.

(v) Let $v$ be a non-strictly convex norm on $\mathbb{R}^{2}$ whose dual ball has extreme points as a proper subset of the unit circle. Let $E_{1}$ be $\mathbb{R}^{2}$ with Euclidean norm and let $E_{2}$ be $\mathbb{R}^{2}$ with the norm $\nu$. Let $V(1)=V(2)$ be a nice linear operator from $E_{1}$ to $E_{2}$ (for example, the identity), and let $\varphi$ be a permutation of $\{1,2\}$. Then $T F(t)=V(t) F(\varphi(t))$ defines 
a nice isomorphism from $X=C\left(\{1,2\}, E_{1}\right)$ onto $Y=C\left(\{1,2\}, E_{2}\right)$ which is not an isometry and for which $T^{*}\left(\operatorname{ext}\left(Y^{*}\right)\right) \neq \operatorname{ext}\left(X^{*}\right)$.

(vi) If, in the statement of Theorem 2.9, we assume that $T^{-1}$ is also nice and $Z\left(E_{1}\right)$ is trivial, we can conclude that $\varphi$ is a homeomorphism. Simply apply Theorem 2.9 to $T^{-1}$ to conclude that $\varphi$ is one-to-one with a continuous inverse. This gives Behrends' Theorem 1.1.

\section{Nice operators on subspaces of $C_{0}(K, E)$}

A bounded linear operator $T$ from $C_{0}(Q, X)$ into $C_{0}(K, Y)$ is said to be a generalized weighted composition operator[12] if there is a subset $K_{1}$ of $K$, a continuous function $\varphi$ from $K_{1}$ onto $Q$ and a continuous operator valued map $V$ from $K_{1}$ into the space of bounded operators from $X$ to $Y$ (with the strong operator topology) such that $T F(t)=V(t) F(\varphi(t))$ for $t \in K_{1}$. A theorem of Cambern [6] shows that if the Banach space $Y$ is strictly convex, then any isometry $T$ as above is a generalized weighted composition operator. Moreover, it has been pointed out by Jeang and Wong [12] that, at least in the case of real spaces, if every isometry $T$ from $C_{0}(Q, X)$ into $C_{0}(K, Y)$ is a generalized weighted composition operator for any $Q, X, K$, then $Y$ must be strictly convex.

In light of this, we can ask what can be said in case $Y$ is not strictly convex. As in the previous section, we wish to make use of extreme point methods, and so we will investigate what happens with nice operators. Since isometries are nice, we accomplish some new results for isometries as well. Following the methods of Novinger [17] in the scalar case, we are led to consider an extension of the notion of Choquet Boundary, usually defined for subspaces of a $C(K)$-space.

DEFINITION 3.1. Let $K$ be a locally compact Hausdorff space, and $N$ a closed subspace of $C_{0}(K, E)$ where $E$ is a Banach space. An element $t \in K$ is in the Choquet Boundary of $N$, written $\operatorname{ch}(N)$, if there exists $x^{*} \in \operatorname{ext}\left(E^{*}\right) \operatorname{such}$ that $x^{*} \circ \psi_{t}$ is an extreme point of the unit ball of $N^{*}$.

It can be shown that $\operatorname{ch}(N)$ is a boundary, that is, given any $F \in N$ there exists some $t \in \operatorname{ch}(N)$ such that $\|F(t)\|=\|F\|$. The definition we have given is an extension of the one due to Novinger [17]. The little book by Phelps [18] is an excellent reference for information on the classical Choquet Boundary.

The Choquet Boundary in the vector-valued case presents some complications not present in the scalar-valued case. We will illustrate this with some examples. Before giving the examples, we need to introduce some special notation. Given $N, K, E$ as in Definition 3.1, we let $E(t)=\{G(t) \in E: G \in N\}$, and

$$
N^{*}(t)=\left\{x^{*} \in \operatorname{ext}\left(E^{*}\right): x^{*} \circ \psi_{t} \in \operatorname{ext}\left(N^{*}\right)\right\} .
$$


We note that if $E(t)$ is not closed, we can take its closure and not cause any significant changes to arguments involving it. Also, since an extreme point for $B\left(E(t)^{*}\right)$ is the restriction to $E(t)$ of an extreme point of $B\left(E^{*}\right)$, we will not always distinguish between the two.

In the examples below, we will consider the scalars to be real.

EXAMPLE 3.2. . (i) Let $N \subset C\left(\{1,2,3\}, \ell^{2}(2)\right)$ be the collection of functions $F$ such that $F(3)=\left(\left\langle F(1), e_{1}\right\rangle,\left(\left\langle F(1), e_{2}\right\rangle+\left\langle F(2), e_{2}\right\rangle\right) / 2\right)$. (By $e_{1}, e_{2}$ are meant the usual unit vectors, and $\langle\cdot, \cdot\rangle$ is the inner product.) Then $\operatorname{ch}(N)=\{1,2,3\}$, and while $e_{1}^{*} \circ \psi_{3}$ is extreme, $e_{2}^{*} \circ \psi_{3}$ is not. However, $x^{*}=(2 / \sqrt{5}, 1 / \sqrt{5}) \in N^{*}(3)$ so that $N^{*}(3)$ spans $E^{*}(3)=E^{*}$.

(ii) Let $N \subset C\left(\{1,2\}, \ell^{2}(2)\right)$ be the space of functions $G$ such that

$$
G(2)=\left(\left\langle G(1), e_{1}\right\rangle,\left\langle G(1), e_{2}\right\rangle / 2\right) .
$$

Then $\operatorname{ch}(N)=\{1,2\}$ and $N^{*}(2)=\left\{ \pm e_{1}^{*}\right\}$, which does not span $E^{*}(2)=E^{*}$.

(iii) Let $\varphi_{1}, \varphi_{2}$ be continuous functions from a locally compact Hausdorff space $K$ to a compact Hausdorff space $Q$ and suppose there is a proper subset $\Gamma$ of $K$ which is the set of all $t \in K$ such that $\varphi_{1}(t)=\varphi_{2}(t)$, and for which $\varphi_{1}(\Gamma)=Q$. Let $T$ be defined on $C(Q, E)$ into $C_{0}(K, E)$ by $T F(t)=\left[F\left(\varphi_{1}(t)+F\left(\varphi_{2}(t)\right)\right] / 2\right.$ for all $F \in C(Q, E)$. (Here, as usual, $\mathrm{E}$ is a given Banach space.) Then $T$ is an isometry, and if $N$ denotes the range of $T$, we have $\operatorname{ch}(N)=\Gamma$, and $N^{*}(t)=\operatorname{ext}\left(E^{*}(t)\right)=\operatorname{ext}\left(E^{*}\right)$ for all $t \in \Gamma$.

The example in (iii) above is an adaptation of an example given originally by McDonald [15]. Note that $T$ does not have the canonical form for all $t \in K$, but does for $t \in \operatorname{ch}(N)$.

We will see now that the methods used in the proof of Theorem 2.8 can be used in the subspace setting. We begin by characterizing the centralizer of a subspace $N$ of $C_{0}(K, E)$. It requires some special assumptions and for that we need a bit more notation (borrowed from Font [10]). For a closed subspace $A$ of $C_{0}(K)$, we say $N \in \mathscr{A}(A)$ if for every $u \in E$ and $h \in A$, the function $h(\cdot) u \in N$.

LEMMA 3.3. Let $N$ be a closed subspace of $C_{0}(K, E)$ with $\operatorname{dim} Z(E(t))=1$ for every $t \in \operatorname{ch}(N)$. Suppose further that either

(i) $N^{*}(t)=\operatorname{ext}\left(E(t)^{*}\right)$ for each $t \in \operatorname{ch}(N)$ or

(ii) the linear span of $N^{*}(t)$ is dense in $E(t)^{*}$ for each $t \in \operatorname{ch}(N)$ and $N \in \mathscr{A}(A)$, where $A$ is norming for $K$.

Then for each $W \in Z(N)$, there is a scalar-valued function $\tilde{h}$ defined on $\operatorname{ch}(N)$ such that $W G(t)=\tilde{h}(t) G(t)$ for all $t \in \operatorname{ch}(N)$. 
PROOF. If (i) above is satisfied, then the proof follows exactly the argument in the proof of Lemma 2.5. The statements about extreme points in that proof are still valid since $N^{*}(t)=\operatorname{ext}\left(E(t)^{*}\right)$. Suppose then, that (ii) holds. Since $A$ is norming and $N \in \mathscr{A}(A), E(t)=E$ for each $t$. Given $W \in Z(N), t \in \operatorname{ch}(N)$, and $x^{*} \in N^{*}(t)$ we have $W^{*}\left(x^{*} \circ \psi_{t}\right)=a_{W}\left(x^{*}, t\right)\left(x^{*} \circ \psi_{t}\right)$.

We can define $P(t)$ on $E$ by $P(t) u=W G(t)$ for $G \in N$ with $G(t)=u$. The fact that $P(t)$ is well defined follows exactly as in the proof of Lemma 2.5 and by using the condition that the span of $N^{*}(t)$ is dense in $E^{*}$. The boundedness of $P(t)$ is straightforward to show since for any $u \in E$, there is some $F \in N$ with $\|F\|=\|F(t)\|=\|u\|$. We now show that $P(t)$ is $M$-bounded and therefore a multiplier.

Since $W$ is a multiplier, it is $M$-bounded for some bound $\lambda$. Recall that this means there exists $\lambda>0$ such that for every $G \in N, W G$ is contained in every ball which contains $\{\mu G: \mu \in \mathbb{F},|\mu| \leq \lambda\}$. If $P(t)$ is not $\lambda$-bounded, there exist $u, x \in E$ and $r>0$ such that $\|\mu u-x\|<r$ for all $\mu$ with $|\mu| \leq \lambda$ but $\|P(t) u-x\| \geq r$. Since $A$ is norming, there is an $h \in A$ with $1=\|h\|=h(t)$ and the functions $G(\cdot)=h(\cdot) u, H(\cdot)=h(\cdot) x$ are both in $N$. Now $\|\mu G-H\|<r$ for all $|\mu| \leq \lambda$ and since $W$ is $\lambda$-bounded, we must have $\|W G-H\|<r$. However,

$$
\|W G-H\| \geq\|W G(t)-H(t)\|=\|P(t) u-x\| \geq r
$$

by the definition of $G$ and $H$. We conclude that $P(t)$ is $M$-bounded.

The remainder of the proof follows as in Lemma 2.5.

Given a subspace $A$ of $C_{0}(Q)$, we will say that $s \in Q$ is a strong boundary point for $A$ if for each neighbourhood $U$ of $s$, and each $\epsilon>0$, there is a function $h$ in $A$ such that $1=\|h\|=h(s)$ and $|h(r)|<\epsilon$ for all $r \in Q \backslash U$. The set of all strong boundary points will be denoted by $\sigma(A)$. For a subspace $M$ of $C_{0}(Q, E)$, we say that $M$ is an $A$-module if $f F \in M$ for all $f \in A$ and $F \in M$. We are now ready to give theorems for nice operators from a subspace of a $C_{0}(Q, E)$ onto a subspace $N$ such as discussed above.

THEOREM 3.4. Let $T$ be a nice isomorphism from a closed subspace $M$ of $C_{0}\left(Q, E_{1}\right)$ onto a closed subspace $N$ of $C_{0}\left(K, E_{2}\right)$. Assume that $M \in \mathscr{A}(A)$ and that $M$ is an $A$-module for a subspace $A$ of $C_{0}(Q)$ which separates the points of $Q$.

Suppose that $Z\left(E_{2}(t)\right)$ is trivial and that $N^{*}(t)=\operatorname{ext}\left(E_{2}(t)^{*}\right)$ for each $t \in \operatorname{ch}(N)$. Then there exists a function $\varphi$ from $\operatorname{ch}(N)$ into $\operatorname{ch}(M)$ and for each $t \in \operatorname{ch}(N)$ there is a nice operator $V(t)$ from $E_{1}(\varphi(t))$ into $E_{2}(t)$ such that $T F(t)=V(t) F(\varphi(t))$ for all $t \in \operatorname{ch}(N)$ and $F \in M$. The function $\varphi$ is continuous at each $t$ for which $\varphi(t) \in \sigma(A)$ and its range is dense in $\sigma(A)$. In particular, if $\sigma(A)$ is dense in $Q$, then the range of $\varphi$ is dense in $Q$. 
Proof. Given $t \in \operatorname{ch}(N)$ and $y^{*} \in N^{*}(t)$ there exists $s \in \operatorname{ch}(M)$ and $x^{*} \in$ $\operatorname{ext}\left(X(s)^{*}\right)$ such that $T^{*}\left(y^{*} \circ \psi_{t}\right)=x^{*} \circ \psi_{s}$. This holds because $T$ is nice. We define $\varphi(t)=s$ and because of Lemma 3.3 we can show that $\varphi$ is well defined with the same argument used for that purpose in the proof of Theorem 2.8. The definition of $V(t)$ and the fact that it is well-defined, bounded, and nice can also be proved as before. If $\varphi(t) \in \sigma(A)$, then we can find the necessary function $F \in M$ so that the continuity of $\varphi$ follows as in the proof of Theorem 2.9, with the exception that we get $\liminf \left\|T F\left(\varphi\left(t_{\beta}\right)\right)\right\| \leq \epsilon$ rather than zero.

It remains to prove the assertion about the density of $\varphi(\operatorname{ch}(N))$. Suppose that $s \in \sigma(A)$ is not in the closure of $\varphi(\operatorname{ch}(N))$. For any positive integer $n$, there is an $h_{n} \in A$ such that $\left\|h_{n}\right\|=h_{n}(s)=1$ and $\left|h_{n}(r)\right|<1 / n$ for all $r \in \varphi(\operatorname{ch}(N))^{-}$. If $u \in E_{1}$ with $\|u\|=1$, let $F_{n}=h_{n} \cdot u$. Then $F_{n} \in M$ and

$$
\left\|T F_{n}(t)\right\|=\left\|V(t) F_{n}(\varphi(t))\right\| \leq\left\|F_{n}(\varphi(t))\right\|<1 / n
$$

for all $t \in \operatorname{ch}(N)$. It follows that $\left\|T F_{n}\right\|<1 / n$ because $\operatorname{ch}(N)$ is a boundary for $N$. Since each $F_{n}$ has norm 1 , this would imply that $T^{-1}$ is unbounded which is a contradiction.

In the previous theorem, we assumed that $N$ satisfied condition (i) of Lemma 3.3. If we assume that $N$ satisfies condition (ii) of that lemma, we get a slightly weaker conclusion; namely, we can no longer prove that $V(t)$ is nice.

THEOREM 3.5. Assume that $M$ has the same properties as in Theorem 3.4, and that $T$ is a nice isomorphism from $M$ onto $N \subset C_{0}\left(K, E_{2}\right)$. Suppose that $Z\left(E_{2}(t)\right)$ is trivial, the linear span of $N^{*}(t)$ is dense in $E_{2}(t)^{*}$ for each $t \in \operatorname{ch}(N)$, and $N \in \mathscr{A}(B)$ where $B$ is norming for $K$ as a subspace of $C_{0}(K)$. Then there exists a function $\varphi$ from $\operatorname{ch}(N)$ into $\operatorname{ch}(M)$ and for each $t \in \operatorname{ch}(N)$ there is an operator $V(t)$ from $E_{1}(\varphi(t))$ to $E_{2}(t)$ such that $T F(t)=V(t) F(\varphi(t))$ for all $t \in \operatorname{ch}(N)$ and $F \in M$. The function $\varphi$ has range dense in $\sigma(A)$. It is continuous and the operator $V(t)$ is a contraction for each $t$ such that $\varphi(t) \in \sigma(A)$. If $\sigma(A)$ is dense in $Q$, then the range of $\varphi$ (and so also, $\operatorname{ch}(M))$ is dense in $Q$.

Proof. We get $\varphi$ well defined as in the previous theorem. Again, for $t \in \operatorname{ch}(N)$ and $u \in E_{1}(\varphi(t))$, we define $V(t) u=T F(t)$ where $F \in M$ such that $F(\varphi(t))=u$. If $H \in M$ with $H(\varphi(t))=u$, we can easily show that $y^{*}(T F(t))=y^{*}(T H(t))$ for all $y^{*}$ in the linear span of $N^{*}(t)$. We now use the density of such functionals in $E_{2}(t)$ to show that the above equality holds for all $y^{*} \in E_{2}(t)^{*}$. If $\varphi(t) \in \sigma(A)$, and $u \in E_{1}(\varphi(t))$, there exists $f \in A$ such that $1=f(\varphi(t))=\|f\|$ and $F=f(\cdot) u \in M$. Thus $\|V(t) u\|=\|V(t) F(\varphi(t))\|=\|T F(t)\| \leq\|F\|=\|u\|$. Hence, $V(t)$ is a contraction. The asserted continuity of $\varphi$ and density of its range follows as in the previous theorem. 
REMARKS. (i) The condition that $A$ separates the points of $Q$ in both Theorems 3.4 and 3.5 could be weakened slightly by supposing that $A$ separates the points of $\operatorname{ch}(M)$. Similarly, it would be enough, in the statement of Lemma 3.3 (ii), and in the statement of Theorem 3.5, to assume that $A$ or $B$ are norming for $\operatorname{ch}(N)$. If in Example 3.2 (iii), we take $K=[0,1]=Q, \Gamma=[0,1 / 2]$ and assume that $\varphi_{1}$ is one-to-one on $\Gamma$, then for $A=\left\{h \in C(K): h \circ \varphi_{1}=h \circ \varphi_{2}\right\}$, the space $N$ will be in $\mathscr{A}(A)$, where $A$ is norming for $\operatorname{ch}(N)=\Gamma$.

(ii) A subspace $A$ of $C_{0}(Q)$ is said to be extremely regular if $\sigma(A)=Q$. It is shown in [7] that such subspaces arise, for example, as kernels of nonzero, continuous complex-valued finite regular Borel measures on $Q$, and that $C_{0}(Q)$ has proper extremely regular subspaces whenever $Q$ is not dispersed. It is also known that if $A$ is a point-separating closed subalgebra of $C_{0}(Q)$, then the strong boundary of $A$ is dense in the Šilov boundary of $A$ [10], which itself is dense in $Q$ if $A$ is a regular closed subalgebra.

(iii) If $A$ is extremely regular, or if it is a regular closed subalgebra of $C_{0}(Q)$, then for $M$ as in the hypotheses of either Theorem 3.4 or Theorem 3.5, we would have $\varphi(\operatorname{ch}(N))$ (and therefore also $\operatorname{ch}(M))$ dense in $Q$.

(iv) Obviously, $M=C_{0}\left(Q, E_{1}\right)$ satisfies all the required hypotheses for $M$. Theorem 3.4 applies, in this case, to the interesting example given by Cambern [6]. In this example, Cambern constructs an isometry from $C\left(\{1,2\}, \ell^{2}\right)$ into $C\left(K, \ell^{2}\right)$, where $K$ is the one point compactification of the positive integers $\mathbb{N}$, for which $\operatorname{ch}(N)=\mathbb{N}$ and the function $\varphi$ cannot be extended continuously to $K$.

(v) The methods of this section can be easily applied to show that the conclusions of both Theorem 3.4 and Theorem 3.5 hold if $M$ is replaced by a function module $\left(Q,\left(X_{s}\right)_{s \in Q}, X\right)$.

(vi) The reader may also wish to compare the conditions given by Font $[10$, Theorem 1] for isometries from $M$ onto $N$. The map corresponding to $\varphi$ in his case goes from a set $K_{0} \subset K$ into $\sigma(A)$. This set $K_{0}$ is not necessarily the same as $\operatorname{ch}(N)$ as we will see in an example later. The assumption there about $M$ is that $M \in \mathscr{A}(A)$ where $A$ is regular. (Font defines $A$ to be regular if for each closed subset $C$ of $Q$ and $s \in Q \backslash C$,there is $h \in A$ with $h(s)=1$ and $h \equiv 0$ on $C$.) The space $E_{2}$ is assumed to be strictly convex and no other conditions are put on $N$.

We will end this section with several examples. First, we want to make a rather special observation. Note that in Example 3.2, part (iii), we have a subspace $N$ of $C(K, E)$ which is defined as the range of a certain isometry with the canonical form on the subset $\Gamma=\operatorname{ch}(N)$. Since $N^{*}(t)=\operatorname{ext}\left(E^{*}\right)$ for each $t \in \operatorname{ch}(N)$, it will follow from Theorem 3.4 that if $E(t)$ has trivial centralizer for each $t \in \operatorname{ch}(N)$ then any nice isomorphism $T$ from any $C_{0}\left(Q, E_{1}\right)$ onto $N$ will be a generalized weighted composition operator. That is, if there is a canonical isometry with range $N$ and $E(t)$ has trivial centralizer for each $t$, then every nice isomorphism onto $N$ will be 
canonical. We state this formally as follows.

THEOREM 3.6. Suppose there exists an isometry $T$ from $C_{0}\left(Q, E_{1}\right)$ onto a subspace $N$ of $C_{0}(K, E)$ so that $T F(t)=V(t) F(\varphi(t))$ for all $t \in \operatorname{ch}(N)$ where $\varphi$ is continuous and $V(t)$ is nice for each $t \in \operatorname{ch}(N)$. Suppose further that $E(t)$ has trivial centralizer for each $t \in \operatorname{ch}(N)$. If $L$ is any locally compact Hausdorff space, $E_{2}$ is any Banach space and $S$ is a nice isomorphism from $C_{0}\left(L, E_{2}\right)$ onto $N, S$ will be a generalized weighted composition operator whose operator weights are nice.

PROOF. Given $t \in \operatorname{ch}(N)$ and $y^{*} \in \operatorname{ext}\left(E(t)^{*}\right)$, since $V(t)$ is nice, there exists $x^{*} \in \operatorname{ext}\left(E_{1}^{*}\right)$ such that $V(t)^{*} y^{*}=x^{*}$. So $x^{*} \circ \psi_{\varphi(t)} \in \operatorname{ext}\left(C_{0}\left(Q, E_{1}\right)^{*}\right)$ and since $T$ is an isometry, $y^{*} \circ \psi_{t}=\left(T^{*}\right)^{-1}\left(x^{*} \circ \psi_{\varphi(t)}\right)$ is an extreme point for $B\left(N^{*}\right)$. Then $N^{*}(t)=\operatorname{ext}\left(E(t)^{*}\right)$, and the theorem follows from Theorem 3.4.

The following example shows that the set $K_{0}$ mentioned in (vi) above can differ from $\operatorname{ch}(N)$.

EXAMPLE 3.7. Let $E$ be two dimensional real space with norm

$$
\|(a, b)\|_{o}= \begin{cases}|a|+|b|(\sqrt{2}-1) & \text { if }|b| \leq|a| \\ |b|+|a|(\sqrt{2}-1) & \text { if }|a| \leq|b| .\end{cases}
$$

The dual norm is given by

$$
\|(\alpha, \beta)\|_{d}= \begin{cases}|\alpha| & \text { if }|\beta| \leq(\sqrt{2}-1) \\ (|\alpha|+|\beta|) / 2 & \text { if }(\sqrt{2}-1) \leq|\beta| \leq(\sqrt{2}+1)|\alpha| \\ |\beta| & \text { if }(\sqrt{2}+1)|\alpha| \leq|\beta|\end{cases}
$$

Let $N=\operatorname{sp}\left\{G_{1}, G_{2}\right\}$ where $G_{1}, G_{2}$ are elements of $C_{0}(\{1,2,3\}, E)$ defined by

$$
\begin{array}{lll}
G_{1}(1)=e_{1}, & G_{1}(2)=-e_{1}, & G_{1}(3)=-e_{1}, \\
G_{2}(1)=e_{2}, & G_{2}(2)=e_{1}, & G_{2}(3)=-e_{1} .
\end{array}
$$

Then for $G=a G_{1}+b G_{2} \in N$, we have $\|G\|=|a|+|b|$. Also $\operatorname{ch}(N)=\{2,3\}$, $E(t)=\operatorname{sp}\left\{e_{1}\right\}$ for $t \in \operatorname{ch}(N)$, and $\operatorname{ext}\left(E(t)^{*}\right)=N^{*}(t)$ for $t \in \operatorname{ch}(N)$. The operator $T$ from $C\left(\{1\}, \ell^{1}(2)\right)$ to $N$ defined by $T F=\left\langle F(1), e_{1}\right\rangle G_{1}+\left\langle F(1), e_{2}\right\rangle G_{2}$ is an isometry. In fact, it has the canonical form with $\varphi$ defined on all of $\{1,2,3\}$, which is the set $K_{0}=B(T)$ as defined by Font [10] (and also Cambern [6]).

Let $W$ be defined on $C(\{1,2\})$ to $N$ by

$$
W f=\left[\frac{f(1)+f(2)}{2}\right] G_{1}+\left[\frac{f(1)-f(2)}{2}\right] G_{2} .
$$

Here, $W$ is an isometry with canonical form where $\varphi$ is defined only on $\operatorname{ch}(N)=K_{0}$. 
The space $E$ in the example is not strictly convex, and the isometries have the form guaranteed by Theorem 3.4. In fact, the operators act in the same way even if we replace $E$ by $\ell^{2}(2)$. Note that the set $K_{0}=B(T)$ is dependent on the operator $T$, while $\operatorname{ch}(N)$, of course, depends on $N$.

Before giving the next example, we wish to observe that if $T$ is a generalized weighted composition operator, then

$$
F(\varphi(t))=0 \Rightarrow T F(t)=0
$$

for any $t$ in the domain of the function $\varphi$. Jeang and Wong [12] have observed that if $E$ is real and not strictly convex, there exist norm-one elements $u_{1}, u_{2}$ in $E$ such that

$$
\left\|\alpha u_{1}+\beta u_{2}\right\|=\alpha+\beta, \quad \text { for all } \alpha, \beta \geq 0 .
$$

Then the operator $T$ from $C(\{1,2\}, \mathbb{R})$ into $C(\{1,2\}, E)$ by

$$
\begin{aligned}
& T f(1)=\left(\frac{f(1)+f(2)}{2}\right) u_{1}+\left(\frac{f(1)-f(2)}{2}\right) u_{2}, \\
& T f(2)=-\left(\frac{f(1)+f(2)}{2}\right) u_{1}+\left(\frac{f(1)-f(2)}{2}\right) u_{2}
\end{aligned}
$$

is an isometry.

The next two examples show that the conclusion of Theorem 3.4 and Theorem 3.5 can fail if either the density of the linear span of $N^{*}(t)$ or the triviality of $Z\left(E_{2}(t)\right)$ is not satisfied.

EXAMPLE 3.8. Let $E$ be the space $\mathbb{R}^{2}$ with norm determined by the unit ball which is the convex set bounded by the unit circle except that the arcs of the circle in the first and third quadrants are replaced by the line segments connecting $(0,1),(1,0)$ and $(-1,0),(0,-1)$ respectively. Then $e_{1}=(1,0), e_{2}=(0,1)$ are elements of $E$ satisfying (7). Let $T$ be defined as above, and $N$ be the range of $T$. It can be shown that $(1,1) \circ \psi_{1},(1,1) \circ \psi_{2}$ are extreme points for the unit ball of $N^{*}$ so that $\operatorname{ch}(N)=\{1,2\}$. However, $N^{*}(1)=N^{*}(2)=\{(1,1),(-1,-1)\}$ which does not span $E^{*}$. The isometry $T$ is not a generalized weighted composition operator because it does not satisfy (6). In this case, the function $\varphi$ could be defined, but $V(t)$ cannot be defined correctly since $N^{*}(1)$ and $N^{*}(2)$ are not big enough. The space $E$ does have trivial centralizer since it has no $M$-summands.

EXAMPLE 3.9. Let $E$ be the real space $\ell^{1}(2)$ and define $T$ as in the previous example. Here we have

$$
T^{*}\left((1,1) \circ \psi_{1}\right)=\psi_{1} \quad \text { and } \quad T^{*}\left((-1,1) \circ \psi_{1}\right)=-\psi_{2}
$$

and the function $\varphi$ is not well-defined. Of course, the centralizer of $E$ is not trivial in this case. 


\section{The strictly convex case}

A Banach space $E$ is strictly convex if every element on the surface of the unit ball is an extreme point. This property has been part of the hypotheses in many of the vector-valued Banach-Stone Theorems, including the first such theorem by Jerison [13] as well as in the previously cited papers of Cambern [6] and Font [10]. The presence of this assumption allows a different approach and we want to show how that goes for nice operators. There are only slight differences from what has been done for isometries by Font. The lemma below is the key. We do it first for a nice isomorphism defined on a function module and the basic ideas in this setting actually go back to Cambern [6].

LEMMA 4.1. Let $X=\left(Q,\left(X_{s}\right)_{s \in Q}, X\right)$ be a function module with the property that each $X_{s}$ is reflexive. Suppose that $T$ is a nice isomorphism from $X$ onto $N \subset C_{0}(K, E)$, where $E$ is strictly convex. Suppose that $y^{*} \circ \psi_{t} \in \operatorname{ext}\left(N^{*}\right)$ and $T^{*}\left(y^{*} \circ \psi_{t}\right)=x^{*} \circ \psi_{s}$. If $F \in X$ and $F(s)=0$, then $T F(t)=0$.

Proof. We begin by assuming $F \in X$ vanishes on a neighbourhood $U$ of $s \in Q$, and also that $\|F\|<1$. Since $X_{s}$ is reflexive, there exists $u \in X_{s}$ such that $x^{*}(u)=$ $\|u\|=1$. Furthermore, by Lemma 2.3 , there exists $F_{1} \in X$ such that $\left\|F_{1}\right\|=1$, $F_{1}(s)=u$, and $\left\|F_{1}(r)\right\|=0$ if $r \in Q \backslash U$. Let $G=F+F_{1}$ and $H=\left[G+F_{1}\right] / 2$. Now $G(s)=H(s)=F_{1}(s)=u$ and each of the functions has norm one. Moreover,

$$
\begin{aligned}
1 & =y^{*}\left(T F_{1}(t)\right)=x^{*}\left(F_{1}(s)\right) \\
& =x^{*}(H(s))=y^{*}(T H(t))=x^{*}(G(s))=y^{*}(T G(t)) .
\end{aligned}
$$

Since $T$ is nice, and therefore a contraction, we conclude that

$$
\|T G(t)\|=\|T H(t)\|=\left\|T F_{1}(t)\right\|=1 .
$$

Note that $T H(t)$ is a proper convex combination of the other two, and since all lie on the surface of the unit ball of the strictly convex space $E$, they must all be equal to each other. Since $T G(t)=T F(t)+T F_{1}(t)$, we must conclude that $T F(t)=0$.

Suppose $F(s)=0$ and let $\epsilon>0$ be given. Since the map $s \rightarrow\|F(s)\|$ is upper semicontinuous, there is a neighbourhood $U$ of $s$ such that $\|F(r)\|<\epsilon$ for all $r \in U$. There exists a continuous real function $g$ on $Q$ of norm one such that $g=0$ on a closed neighbourhood $V$ of $s$ which is contained in $U$ and which has the value 1 on the closed set $Q \backslash U$. Then $g F \in X$ vanishes on a neighborhood of $s$ and $\|F-g F\|<2 \epsilon$. By the first part of the argument, we have $T(g F)(t)=0$ and therefore,

$$
\|T F(t)\|=\|T F(t)-T(g F)(t)\| \leq\|T F-T(g F)\| \leq\|F-g F\|<2 \epsilon .
$$

It follows that $T F(t)=0$. 
Font [10] gives a similar result for isometries on certain subspaces $M$ of $C_{0}\left(Q, E_{1}\right)$. We state the lemma for the case of nice operators where $M$ is a $C_{0}(Q)$-module which is norming. We seem to need this condition in order to be sure that the functions that vanish on a neighbourhood of $s$ are dense in the functions that vanish at the single point.

LEMMA 4.2. Let $M$ be a subspace of $C_{0}\left(Q, E_{1}\right)$ which is norming for $Q$ (or even just for $\operatorname{ch}(M))$, where $E_{1}$ is reflexive and suppose $M$ is a $C_{0}(Q)$-module. Suppose that $T$ is a nice isomorphism from $M$ onto $N \subset C_{0}(K, E)$, where $E$ is strictly convex. Suppose that $y^{*} \circ \psi_{t} \in \operatorname{ext}\left(N^{*}\right)$ and $T^{*}\left(y^{*} \circ \psi_{t}\right)=x^{*} \circ \psi_{s}$. If $F \in M$ and $F(s)=0$, then $T F(t)=0$.

Proof. Assume that $F$ vanishes on a neighbourhood $U$ of $s$. Since $E_{1}$ is reflexive, there exists $u \in E_{1}$ such that $x^{*}(u)=\|u\|=1$. The norming property of $M$ gives $F_{0} \in M$ such that $F_{0}(s)=u$ and $1=\left\|F_{0}\right\|=\left\|F_{0}(s)\right\|$. Furthermore, there exists $h \in C_{0}(Q)$ with $1=h(s)=\|h\|$ and $h(r)=0$ for $r \in Q \backslash U$, and because $M$ is a $C_{0}(Q)$-module, the function $F_{1}=h F_{0}$ is in $M$ and has the same properties as the $F_{1}$ defined in the beginning of the proof of the previous lemma. The remainder of the proof differs from that of the previous lemma only in the second part. Given that $F(s)=0$, and $\epsilon>0$, there is a neighbourhood $U$ of $s$ and a compact set $D$ such that $\|F(r)\|<\epsilon$ for all $r \in U$ and all $r \in Q \backslash D$. There exists $g \in C_{0}(Q)$ such that $\|g\|=1, g=0$ on a compact neighbourhood $V$ contained in $U$ and $g=1$ on $D \backslash U$. Then $g F \in M$ vanishes on a neighbourhood of $s$, and we complete the argument as before.

The conclusions of the above lemmas are just what is needed to show that a nice operator has the canonical form.

THEOREM 4.3. Let $T$ be a nice isomorphism as in the statement of Lemma 4.1 (alternately, Lemma 4.2). Then there exists a continuous function $\varphi$ from $\operatorname{ch}(N)$ into $Q$ whose range is dense and for each $t \in \operatorname{ch}(N)$, there is a bounded operator $V(t)$ from $X_{\varphi(t)}$ (alternately, $\left.E_{1}\right)$ to $E_{2}(t)$ such that $T F(t)=V(t) F(\varphi(t))$ for all $t \in \operatorname{ch}(N)$.

Proof. Let $t \in \operatorname{ch}(N)$ and suppose

$$
T^{*}\left(y^{*} \circ \psi_{t}\right)=x^{*} \circ \psi_{s} ; \quad T^{*}\left(z^{*} \circ \psi_{t}\right)=w^{*} \circ \psi_{r}
$$

Let $u \in X_{r}$ (alternately, $u \in E_{1}$ ) be such that $w^{*}(u) \neq 0$. There exists $F$ with $F(s)=0$ and $F(r)=u$. By Lemma 4.1 (alternately, Lemma 4.2) we have $T F(t)=0$. However, $0 \neq w^{*}(F(r))=\left(w^{*} \circ \psi_{r}\right)(F)=z^{*}(T F(t))=0$. This contradiction shows that there is a well-defined function $\varphi$ which pairs $t$ with $s$. 
For $s=\varphi(t)$ and $H(s)=F(s)$, we have $(H-F)(s)=0$, and by Lemma 4.1 (alternately, Lemma 4.2) again, we get $T(H-F)(t)=0$. We conclude that the equation

$$
V(t) u=T F(t), \quad \text { where } F(s)=u,
$$

describes a well-defined operator from $X_{s}$ to $E_{2}(t)$. The rest of the argument for $T$ as in Lemma 4.1 follows as in earlier proofs. For the case in which $T$ is defined on $M$ as in Lemma 4.2, the proof of continuity and density of the function $\varphi$ depends on the existence of certain functions in $M$. Given $u \in E_{1}, s \in Q$, a neighbourhood $U$ of $s$, and $\epsilon>0$, we can follow the ideas of the construction given in the proof of Lemma 4.2 above to find a function $F \in M$ with $F(s)=u,\|F\|=\|u\|$, and $F(r)=0$ for $r \in Q \backslash U$. The continuity of $\varphi$ and the density of its range can be proved as in the proof of Theorem 3.4.

The result above holds for $T$ defined on $C_{0}\left(Q, E_{1}\right)$ onto $N$. This is the theorem of Cambern [6] for nice operators instead of isometries. Of course we have had to assume that $E_{1}$ is reflexive.

REMARKS. (i) The assumption that $E_{2}$ is strictly convex implies that $Z\left(E_{2}(t)\right)$ is trivial for every $t \in \operatorname{ch}(N)$. However, we get the conclusion of Theorem 4.3 without any of the other assumptions about $N$ needed in the Theorems in Section 3. Of course, we did have to assume the reflexivity condition on $E_{1}$.

(ii) The map $t: \rightarrow V(t)$ of Theorem 4.3 (the alternate case) can be proved to be continuous from $\operatorname{ch}(N)$ into $\mathscr{L}\left(E_{1}, E\right)$ with the S.O.T provided that for each $u \in E_{1}$ there is a function $F \in M$ which is constantly equal to $u$ on a neighbourhood of $t$. This would hold, for example, if $M \in \mathscr{A}(A)$ where $A$ is normal. (A subspace $A$ is normal if given a pair of disjoint compact sets, there is a function in $A$ which is identically 1 on one of the sets and zero on the other.) This remark is also pertinent for the theorems of Section 3. It is known that a closed regular subalgebra $A$ of $C_{0}(Q)$ is normal [14].

(iii) As we mentioned before, Lemma 4.2 is given by Font [10] for $T$ an isometry, but Font only assumes that $M \in \mathscr{A}(A)$ where $A$ is a regular subspace of $C_{0}(Q)$. It is argued there that under these conditions, the set of functions that vanish in a neighbourhood of a point $s$ in the strong boundary of $A$ is dense in the set of functions that vanish at $s$. We were unable to prove that assertion. Our proof of this fact seems to need a slightly stronger condition than normality for $A$. It is tempting to define $A$ to be strongly normal if for any disjoint pair of compact sets there is an element of $A$ which has norm 1 (or at least some uniform bound on the norm) which is zero on one compact set and 1 on the other. However, it is known that such a subspace of $C_{0}(Q)$ is necessarily all of $C_{0}(Q)$ [14, page 178]. 


\section{References}

[1] H. Al-Halees, Banach-Stone theorems for nice operators on Banach function modules (Ph.D. Thesis, Central Michigan University, 2001).

[2] S. Banach, Theorie des operations lineares (Chelsea, Warsaw, 1932).

[3] E. Behrends, $M$ structure and the Banach-Stone theorem, Lecture Notes in Math. 736 (Springer, Berlin, 1979).

[4] B. Brosowski and F. Deutsch, 'On some geometric properties of suns', J. Approx. Theory 10 (1974), 245-267.

[5] M. Cambern, 'On mapping of spaces of functions with values in a Banach space', Duke Math. $J$. 48 (1975), 91-98.

[6] _ 'A Holsztynski theorem for spaces of vector valued functions', Studia Math. 63 (1978), 213-217.

[7] B. Cengiz, 'On extremely regular function spaces', Pac. J. Math. 49 (1973), 335-338.

[8] N. Dunford and J. Schwartz, Linear operators, Part I (Interscience, New York, 1958).

[9] R. Fleming and J. Jamison, 'Isometries on Banach spaces: a survey', in: Analysis, geometry, and groups, a Riemann legacy volume (eds. H. Srivastava and T. Rassias) (Hadronic Press, Palm Harbor, Florida, 1993) pp. 52-123.

[10] J. Font, 'Linear isometries between certain subspaces of continuous vector-valued functions', Illinois J. Math. 42 (1998), 389-397.

[11] W. Holsztyński, 'Continuous mappings induced by isometries of spaces of continuous functions', Studia Math. 26 (1966), 133-136.

[12] J. Jeang and N. Wong, 'On the Banach-Stone problem', preprint, 2001.

[13] M. Jerison, 'The space of bounded maps into a Banach space', Ann. of Math. 52 (1950), 309-327.

[14] G. Leibowitz, Lectures on complex function algebras (Scott, Foresman, and Company, Glenview, Illinois, 1970).

[15] J. McDonald, 'Isometries of function algebras', Illinois J. Math. 17 (1973), 579-583.

[16] P. Morris and R. Phelps, 'Theorems of Krein-Milman type for certain convex sets of operators', Trans. Amer. Math. Soc. 150 (1970), 183-200.

[17] W. Novinger, 'Linear isometries of subspaces of continuous functions', Studia Math. 53 (1975), 273-276.

[18] R. Phelps, Lectures on Choquet's theorem, Lecture Notes in Math. 1757, 2nd Edition (Springer, Berlin, 2001).

[19] M. H. Stone, 'Applications of the theory of boolean rings in topology', Trans. Amer. Math. Soc. 41 (1937), 375-481.

[20] D. Werner, 'Extreme points in spaces of operators and vector-valued measures', Proc. Amer. Math. Soc. 89 (1983), 1-9.

Department of Mathematics

Saginaw Valley State University

Saginaw MI 48710

USA

e-mail: hhalees@svsu.edu
Department of Mathematics

Central Michigan University

Mt. Pleasant MI 48859

USA

e-mail: flemilrj@cmich.edu 